\title{
Transmitted Energy as a Basic System Resource
}

\author{
Aarne Mämmelä ${ }^{1)}$, Ilkka Saarinen ${ }^{1)}$, Desmond P. Taylor ${ }^{2)}$ \\ ${ }^{1)}$ VTT Electronics, P.O. Box 1100, FI-90571 Oulu, Finland \\ ${ }^{2)}$ University of Canterbury, Private Bag 4800, Christchurch, New Zealand
}

\begin{abstract}
Energy is a basic resource in digital transmission links. Physically, radio channels correspond to passive circuits and most of the transmitted energy is lost in the channel. Two alternative approaches are used for performance measurements in terms of energy. Either the average transmitted or received energy per bit is used, both usually normalized by the receiver noise spectral density. This leads to the average transmitted or received signal-to-noise ratio (SNR) per bit, respectively. However, the transmitted energy is the basic system resource. The average energy gain of a channel depends on the transmitted signal. For convenience, the transmitted SNR referred to the receiver is defined to be the product of the transmitted SNR and the representative energy gain, which is defined as the average energy gain of a signal that is uniformly distributed in all dimensions: time, frequency and space. An explicit relationship between the transmitted and received SNR's using the covariance concept is derived. Limitations of the use of different SNR definitions are summarized.
\end{abstract}

Keywords-transmitted signal-to-noise ratio; transmitted signalto-noise ratio referred to the receiver; received signal-to-noise ratio; multipath fading

\section{INTRODUCTION}

Energy is a basic resource in digital transmission links [1], [2] and we are interested in how efficiently it is used. There appear to be two alternative ways for performance measurements in terms of energy. Either the average transmitted energy [3] - [7] or average received energy [8] [10] per bit is used, both usually normalized by the receiver noise spectral density, leading to the average transmitted and received signal-to-noise ratio (SNR) per bit, respectively.

Transmitted energy is the basic system resource since it is the transmitted energy rather than the received energy that is taken from the battery and is necessarily limited. Therefore, the transmitted SNR for a given performance, for example bit error rate (BER), shows how well the transmitter and receiver are jointly "matched" to the channel.

It is well known that the system performance depends partially on the average received SNR. If we compare different receivers for the same transmitted signal and for the same channel, we are able to measure how well the receiver is matched to the channel. However, we do not necessarily know how well the transmitted signal is matched to the channel since the channel attenuation and distortions are included in the received signal. If the channel is selective in time, frequency or space, it may happen that two systems have different transmitted energies although the received energies are identical. The authors of [9] and [10] optimize the use of the transmitted energy in adaptive transmission in a fading channel, but in their results they show how efficiently the received energy is used. The reader is led to believe that the system is almost as good as if there were no fading at all although much improvement is actually possible.

All physical systems follow the energy conservation law. In the radio channel a major part of the transmitted energy is transformed into other forms and lost. Only a small part of the transmitted energy is received due to the finite aperture of the antennas. Electrically a wireless channel corresponds to a passive circuit. To extend the coverage we may use for example relay stations that would make the overall link active. In passive systems the average received SNR is smaller than or equal to the average transmitted SNR. The path loss of the wireless channel is usually very large, for example in the order of $100 \mathrm{~dB}$, and fading may have a dynamic range of $40 \mathrm{~dB}$. Thus, for convenience the transmitted SNR is usually referred to the receiver.

In this paper we define the representative energy gain of the channel so that it includes all dimensions when traditionally only time [7] or frequency [11] is separately included. We use this concept to define the transmitted SNR referred to the receiver as in [7]. A novel relationship between the transmitted SNR referred to the receiver and the received SNR is presented by using the covariance concept. Finally, limitations of the different SNR concepts are summarized. At the end some conclusions are made.

\section{SYSTEM MODEL}

Usually, bits are transmitted by using a carrier that is modulated so that $k$ bit blocks are mapped onto $M$-ary symbols $a_{n}$ where $M=2^{k}$ [8], [12]. We assume here quadrature amplitude modulation (QAM). We will transmit a block of $N_{S}=T_{B} / T$ symbols in an interval $T_{B}$ where $T$ is the symbol interval and $N_{S}$ is assumed to be an integer. The interval $T_{B}$ is the data block length and characterizes the delay from the transmitter to the receiver.

The channel is assumed to be randomly time-variant. We use adaptive power control where the energy of each symbol is selected according to the state of the channel (details below). As a special case, a system with no power control is used. All the random processes are assumed to be ergodic so that time averages are equal to statistical averages. A total of $N=k N_{S}$ bits are transmitted with a total average transmitted energy $\bar{E}_{B}=E\left\{E_{B}\right\}$ within an interval $T_{B}$, in a bandwidth $W$, and having a bit error rate $P_{e}$.

We initially consider a single-input single-output (SISO) system with a slowly fading frequency-nonselective channel, 
and later extend the results to frequency-selective channels and multiple-input multiple-output (MIMO) systems having many antennas in the transmitter and receiver. The received complex baseband signal has the form

$$
r(t)=\sum_{n=0}^{N_{s}-1} \sqrt{E_{n, t x}} a_{n} g(t-n T) H(n T)+w(t)
$$

where $E_{n, t x}$ is the energy of the $n$-th transmitted symbol given by the power control (averaged over $a_{n}$ ), $a_{n}$ is the QAM symbol $\left(E\left\{\left|a_{n}\right|^{2}\right\}=1\right), g(t)$ is the symbol waveform assumed to have unit energy $\left(\int_{-\infty}^{\infty}|g(t)|^{2} d t=1\right), H(t)=v(t) \exp [j \theta(t)]$ is the fading gain representing the channel response, $v(t)=|H(t)|$ and $\theta(t)=\arg [H(t)]$ are the magnitude and phase of the fading gain, respectively, and $w(t)$ is the additive white Gaussian noise (AWGN) with a two-sided power spectral density of $N_{0}$. Its autocorrelation function is $E\left\{w(t+\tau) w^{*}(t)\right\}=N_{0} \delta(\tau)$ where $\delta(\tau)$ is the unit impulse function.

By slow fading we mean that $H(t)$ does not significantly change during the transmission of a symbol waveform $g(t)$ so that over the $n$-th symbol interval we can use the approximation $H(t) \approx H(n T)$. Thus the symbol waveform is not distorted in the channel.

We drop the explicit time dependence (the index $n$ ), so the transmitted energy per symbol is denoted by $E_{t x}$ and the received energy per symbol is denoted by $E_{r x}$. With adaptive power control the energy $E_{t x}$ is changed according to the quality of the channel determined by the ratio $\gamma_{H}=\bar{E}_{t x}|H|^{2} / N_{0}$ [12] where $\bar{E}_{t x}=\bar{E}_{B} / N_{s}$, and we have dropped the argument $t$ of $H(t)$. Power control algorithms can, in general, be divided into water filling and truncated channel inversion. If water filling is used the transmitted energy is $E_{t x}=\bar{E}_{t x}\left(1 / \gamma_{0}-1 / \gamma_{H}\right)$ for $\gamma_{H} \geq \gamma_{0}$ and zero otherwise where $\gamma_{0}$ is a cut-off value. If truncated channel inversion is used, the transmitted energy is $E_{t x}=\bar{E}_{t x}\left(\sigma_{0} / \gamma_{H}\right)$ for $\gamma_{H} \geq \gamma_{0}$ and zero otherwise where $\sigma_{0}$ is a constant selected so that the average transmitted energy is $\bar{E}_{t x}$. The cutoff value is $\gamma_{0}=0$ for full channel inversion.

\section{RESULTS}

\section{A. Representative Energy Gain}

A measure of the received signal quality is the instantaneous received SNR per symbol $\gamma_{r x}=E_{r x} / N_{0}=E_{t x}|H|^{2} / N_{0}$ [8]. The average received $S N R$ per symbol is $\bar{\gamma}_{r x}=\bar{E}_{r x} / N_{0}=E\left\{E_{t x}|H|^{2}\right\} / N_{0}$. Since the transmitted energy is the basic system resource, the ratio $\gamma_{t x}=E_{t x} / N_{0}$ is of interest. Its average is the average transmitted SNR per symbol $\bar{\gamma}_{t x}=\bar{E}_{t x} / N_{0}$.

The average energy gain of the channel, i.e., $G=\bar{\gamma}_{r x} / \bar{\gamma}_{t x}=\bar{E}_{r x} / \bar{E}_{t x}$, depends in general on the transmitted signal and the channel. We define the representative energy gain (denoted by $G_{0}$ ) of the channel as the average energy gain for a signal whose energy is uniformly distributed in time. In our frequency-nonselective channel $G_{0}=E\left\{|H|^{2}\right\}$.

Usually it is convenient, although not mandatory, to refer $\bar{\gamma}_{t x}$ to the receiver, and we use the definition transmitted SNR referred to the receiver $\tilde{\gamma}_{t x}=G_{0} \bar{\gamma}_{t x}$ [6], [7]. This is a somewhat arbitrary but a convenient definition. The ratio $\bar{\gamma}_{t x}$ is scaled by $G_{0}$ so that $\tilde{\gamma}_{t x}=\bar{\gamma}_{r x}$ in the special case when $E_{t x}$ and $|H|^{2}$ are uncorrelated. The tilde $(\sim)$ is used in $\tilde{\gamma}_{t x}$ to emphasize that the averaging is done in an exceptional way, i.e., separately for $E_{t x}$ and $|H|^{2}$ although in practice there may be some correlation due to power control. We note that $\bar{\gamma}_{H}=\tilde{\gamma}_{t x}$.

The error rate performance of the receiver depends on $\bar{\gamma}_{r x}$, but also on $k$, the number of bits/symbol, and the energy $E_{t x}$ for each value of $|H|^{2}$. For example, if $E_{t x}$ is a constant, i.e., no power control is used or, alternatively, $|H|^{2}$ is a constant, i.e., the channel is time-invariant, $E_{t x}$ and $|H|^{2}$ are uncorrelated. The average received SNR per symbol can then be written in the exceptional form $\bar{\gamma}_{r x}=\bar{E}_{t x} G_{0} / N_{0}$. The different SNR's are summarized in Fig. 1.

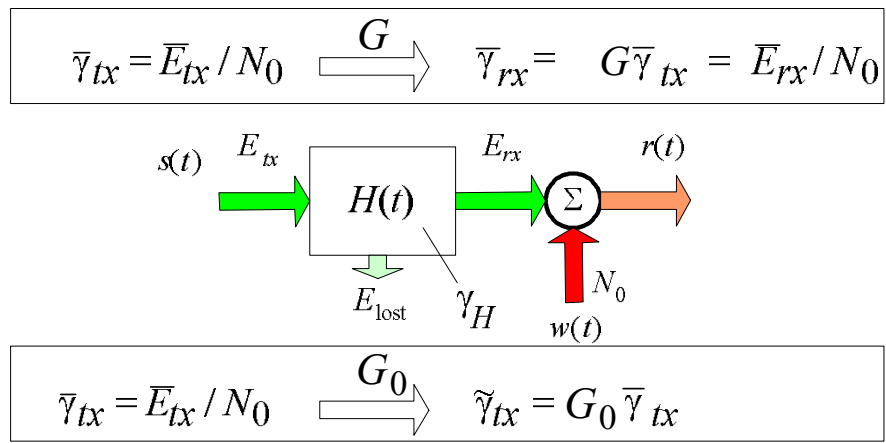

Figure 1. Three different SNR's $\left(s(t)\right.$ is the transmitted signal and $E_{\text {lost }}$ is the energy lost in the channel).

\section{B. Relationship between Transmitted and Received SNR}

The covariance of two random variables $X$ and $Y$ is defined as $C=\operatorname{Cov}(X, Y)=E\{(X-\bar{X})(Y-\bar{Y})\}, \quad$ or equivalently 
$C=E\{X Y\}-E\{X\} E\{Y\}$ where $\bar{X}=E\{X\}$ refers to the mean of $X$. We denote $X=\gamma_{t x}$ and $Y=|H|^{2}$. Therefore

$$
\begin{aligned}
& C=\operatorname{Cov}\left(\gamma_{t x},|H|^{2}\right)=E\left\{\gamma_{t x}|H|^{2}\right\}-E\left\{\gamma_{t x}\right\} E\left\{|H|^{2}\right\} \\
& =\bar{\gamma}_{r x}-\bar{\gamma}_{t x} G_{0}=\bar{\gamma}_{r x}-\tilde{\gamma}_{t x} .
\end{aligned}
$$

It follows that $\bar{\gamma}_{r x}=\tilde{\gamma}_{t x}+C$. We have shown that the average received SNR is equal to the sum of the average transmitted SNR referred to the receiver and the covariance of the instantaneous transmitted SNR and the instantaneous energy gain of the channel.

We will need the following statistical result. If $X$ is a positive random variable with the mean $E\{X\}=\bar{X}$, the inequality $1 / E\{X\} \leq E\{1 / X\}$ is valid. This comes directly from Jensen's inequality for concave functions [13]. The function $1 / x$ is concave for $x>0$ since its second derivative is positive. The equality $1 / E\{X\}=E\{1 / X\}$ is valid only if $X$ is a constant.

If the transmitted signal is uniformly distributed in time, the average energy gain of the channel is equal to the representative energy gain and therefore the covariance is zero by definition $(C=0)$. If water filling is used, we have for $\gamma_{H} \geq \gamma_{0}$

$$
\begin{aligned}
& \bar{\gamma}_{t x} C=\operatorname{Cov}\left\{\gamma_{t x}, \gamma_{H}\right\}=E\left\{\gamma_{t x} \gamma_{H}\right\}-E\left\{\gamma_{t x}\right\} E\left\{\gamma_{H}\right\} \\
& =E\left\{\bar{E}_{t x}\left(1 / \gamma_{0}-1 / \gamma_{H}\right) / N_{0} \cdot \gamma_{H}\right\} \\
& -E\left\{\bar{E}_{t x}\left(1 / \gamma_{0}-1 / \gamma_{H}\right) / N_{0}\right\} E\left\{\gamma_{H}\right\} \\
& =\bar{\gamma}_{t x}\left[E\left\{1 / \gamma_{H}\right\} E\left\{\gamma_{H}\right\}-1\right] \geq 0 .
\end{aligned}
$$

Therefore for water filling $C \geq 0$ and $G \geq G_{0}$. If $\gamma_{H}<\gamma_{0}$, neither symbols nor energy are transmitted.

The interval $T_{B}$ in Section II includes only those time instants for which $\gamma_{H} \geq \gamma_{0}$. The actual time needed to transmit $N_{s}$ symbols, including the time instants when $\gamma_{H}<\gamma_{0}$, is a random variable in a fading channel since $M$ is fixed in our system model. In general water filling is beneficial only when also $M$ is optimized for each symbol interval. Otherwise truncated channel inversion may work better in terms of bit error rate.

For truncated channel inversion for $\gamma_{H} \geq \gamma_{0}$

$$
\begin{aligned}
& \bar{\gamma}_{t x} C=\operatorname{Cov}\left\{\gamma_{t x}, \gamma_{H}\right\}=E\left\{\gamma_{t x} \gamma_{H}\right\}-E\left\{\gamma_{t x}\right\} E\left\{\gamma_{H}\right\} \\
& =E\left\{\bar{E}_{t x}\left(\sigma_{0} / \gamma_{H}\right) / N_{0} \cdot \gamma_{H}\right\} \\
& -E\left\{\bar{E}_{t x}\left(\sigma_{0} / \gamma_{H}\right) / N_{0}\right\} E\left\{\gamma_{H}\right\} \\
& =\bar{\gamma}_{t x} \sigma_{0}\left[1-E\left\{1 / \gamma_{H}\right\} E\left\{\gamma_{H}\right\}\right] \leq 0 .
\end{aligned}
$$

Thus, for truncated channel inversion $C \leq 0$ and $G \leq G_{0}$. As in water filling, if $\gamma_{H}<\gamma_{0}$, neither symbols nor energy are transmitted.

The covariance $C$ is a measure of how well the transmitted signal is "matched" to the channel: the larger the covariance is, the larger the average energy gain $G$ of the channel is. It is well known that water filling maximizes the capacity of the channel implying that the signal is also otherwise optimized. In Fig. 2 we show a qualitative comparison of water filling and truncated channel inversion with no transmitter power control. In the figure the probability density function $f\left(\gamma_{r x}\right)$ of the received SNR is shown in the three cases when $\bar{\gamma}_{t x}$ is fixed. Fig. 2 will be further discussed later.

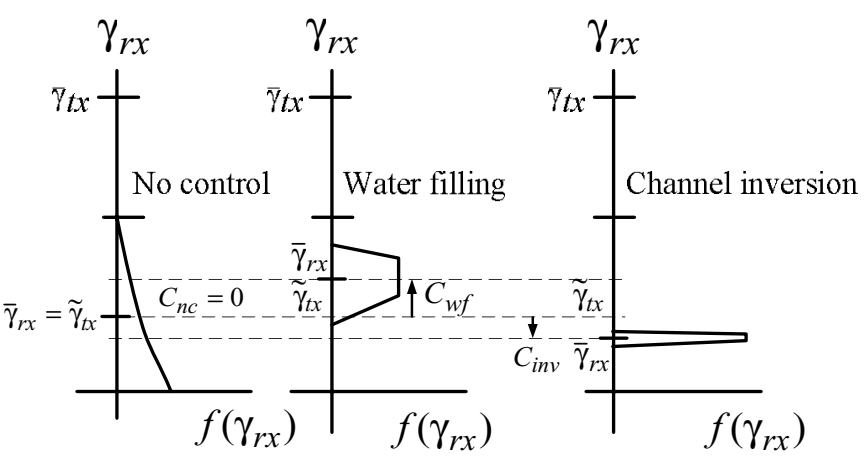

Figure 2. Comparison on water filling (wf) and channel inversion (inv) with no control (nc).

In general, the transmitted SNR $\bar{\gamma}_{t x}$ should be used in comparisons implying that $\bar{E}_{t x}$ is the same in all systems, adaptive or not. The example below elaborates the difference between the transmitted SNR referred to the receiver and received SNR.

Comparison between average transmitted SNR referred to the receiver and received $S N R$ : We consider a simplified pilot symbol system where in each frame, in addition to a data symbol using antipodal modulation $(M=2)$, a pilot symbol for channel estimation purposes is transmitted in a time multiplexed form [14]. The pilot symbol and the antipodal data symbol without power control form an orthogonal symbol set $\{(1,1),(1,-1)\}$. Using power control the energy of the data symbol is adjusted according to the squared value of estimated Rayleigh fading channel attenuation $\hat{v}^{2}(t)$. A Rayleigh fading channel does not strictly speaking correspond to a passive circuit since its instantaneous energy gain can be larger than unity, see [11]. The energy of the pilot symbol is not adjusted. The average energy of the data symbol is constrained to be equal to the energy of the pilot symbol. The power control scheme is designed to minimize the average error rate of the pilot symbol system. The approach resembles the truncated channel inversion scheme [12], but uses a bit error rate criterion. Pilot and data symbols are transmitted all the time, even when the channel is weak since in effect $\gamma_{0}=0$. However, the system is not full channel inversion, which does not in general minimize the bit error rate. 
In Fig. 3 the bit error rate of the pilot symbol system with and without power control is depicted as a function of the average transmitted SNR per bit $\tilde{\gamma}_{t x}$ (energy in the pilot symbol is included) [14]. For comparison, curves for the orthogonal system with optimal power control and the orthogonal system in an AWGN channel having the same average representative energy gain $G_{0}$ are presented. The orthogonal system assumes that the channel is known and the result represents a lower bound on bit error rate for the pilot symbol system with power control. The performance losses of the pilot symbol system using power control compared to the orthogonal system in an AWGN channel are shown for three specific bit error rate levels.

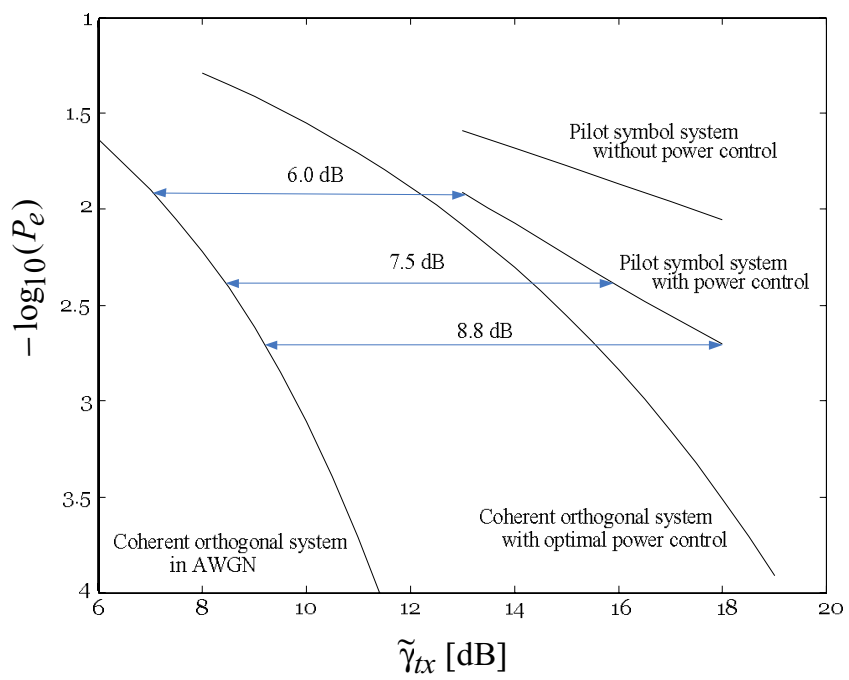

Figure 3. Performance of the pilot symbol system as a function of average transmitted SNR per bit referred to the receiver (correct comparison).

In Fig. 4, the bit error rate of the pilot symbol system with and without power control is plotted now as a function of the average received SNR per bit $\bar{\gamma}_{r x}$ (effect of the pilot symbol is again included) [14]. The performance of the orthogonal system in an AWGN channel is shown for comparison. The pilot symbol system without power control and the orthogonal system in an AWGN channel are in the same positions as in Fig. 3, since in those cases $\bar{\gamma}_{r x}=\widetilde{\gamma}_{t x}$. Again, the performance losses of the pilot symbol system using power control compared to the orthogonal system in an AWGN channel are shown for the same three bit error rate levels. When $\tilde{\gamma}_{t x}=16 \mathrm{~dB}$, the difference between $\tilde{\gamma}_{t x}$ and $\bar{\gamma}_{r x}$ in the pilot symbol system with power control is $5.3 \mathrm{~dB}$. More generally the difference ranges from 4 to $6 \mathrm{~dB}$ between Figs. 3 and 4 . The difference can approach the dynamic range of the channel. From Fig. 4 one could conclude that for example receiver diversity would not give any significant performance gain since the performance is already quite close to the AWGN curve although from Fig. 3 we see that the diversity gain can be significant as shown in [15]. This kind of erroneous conclusion was made in [9], [10] where a very simple power control loop in a fading channel seemingly results in a performance close to that in the AWGN channel. The reason for this behaviour can be seen from Fig. 2. When using $\bar{\gamma}_{r x}$, the covariance $C$ is neglected and the curves are close to each other. In this case apparently the only benefit from power control comes from the better distribution of $\gamma_{r x}$ around the mean $\bar{\gamma}_{r x}$.

\section{Generalizations}

The results can be easily generalized to frequency-selective channels and MIMO systems. Details are omitted. The representative energy gain $G_{0}$ is the average energy gain of a transmitted signal whose energy is uniformly distributed in time, frequency and space. Normally we want to use pulse shaping in the transmitter, for example in the form of square root raised cosine waveform [8]. Therefore we seldom have a uniform distribution in the frequency domain and in general the average energy gain $G$ is not equal to the representative energy gain $G_{0}$ in a frequency-selective channel.

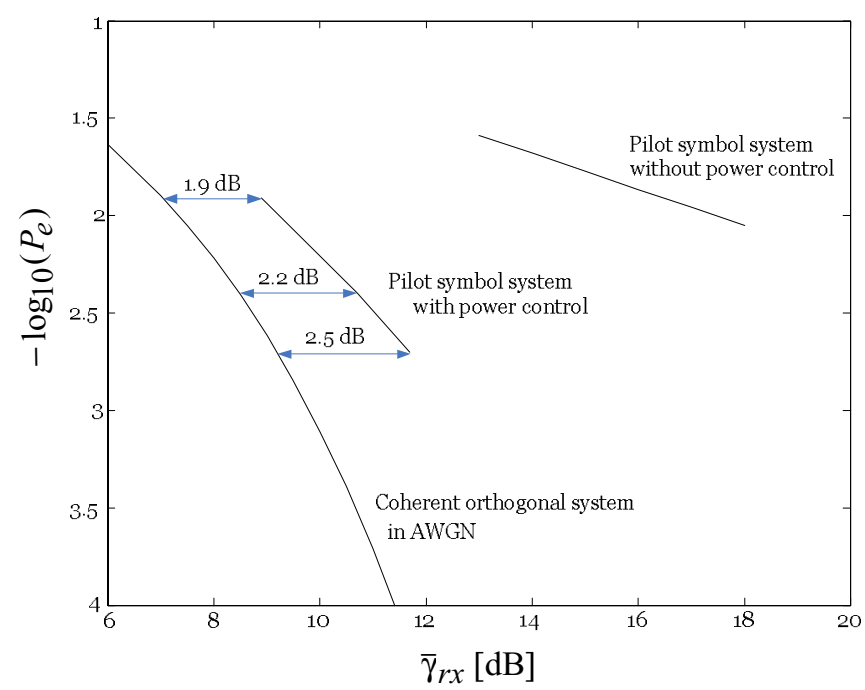

Figure 4. Performance of the pilot symbol system as a function of the average received SNR per bit (misleading comparison).

\section{Use of Different SNR's}

We have defined three different SNR's, namely, the transmitted SNR $\bar{\gamma}_{t x}$, the transmitted SNR referred to the receiver $\tilde{\gamma}_{t x}$, and the received SNR $\bar{\gamma}_{r x}$ (Fig. 1). Since the basic system resource is the transmitted energy, we should use the transmitted SNR in fair comparisons. We can equivalently use the transmitted SNR referred to the receiver $\tilde{\gamma}_{t x}=G_{0} \bar{\gamma}_{t x}$ if we compare different transmitters and receivers in channels with the same $G_{0}$.

We can use the received SNR $\bar{\gamma}_{r x}=G_{0} \bar{\gamma}_{t x}+C$ if and only if both $G_{0}$ and $C$ are fixed in comparisons of different systems. We now assume that $G_{0}$ is a constant so that we can use $\tilde{\gamma}_{t x}=G_{0} \bar{\gamma}_{t x}$ in comparisons. Since $C$ depends on both the transmitted signal and the channel, in general both the transmitter and the channel must be the same in comparisons when using the received SNR. The covariance is $C=0$ when 
the energy of the transmitted signal is uniformly distributed in time, frequency and space, and indeed in this case the received SNR can be used instead of the transmitted SNR referred to the receiver. This is quite a strict requirement that is not usually met, especially in the frequency domain since pulse shaping is usually used. If the channel is an AWGN channel with a gain that does not change in the three domains, $C=0$ and comparisons can be made with the received SNR.

\section{CONCLUSIONS}

The performance of the receiver is defined in part by the received SNR. However, most of the transmitted energy is lost in the wireless channel. Some transmitted signals may be better matched to the channel and therefore they may use the transmitted energy more efficiently than other systems. This is especially valid for adaptive transmission systems. If the received SNR is used in performance comparison, we may end up with misleading conclusions. We have shown that in general the transmitted SNR should be used in fair system comparisons unless there is a specific reason to use the received SNR. The conditions can be summarized as follows: either the energy of the transmitted signal must uniformly distributed in time, frequency and space or the gain of the channel must be a constant in all three dimensions.

\section{ACKNOWLEDGMENT}

This work has been performed in the framework of the IST project IST-2003-507581 WINNER, which is partly funded by the European Union, and the AWICS project, which is partly funded by the Academy of Finland. The authors would like to acknowledge the contributions of their colleagues. Adrian Kotelba is acknowledged for his comments.

\section{REFERENCES}

[1] M. P. Ristenbatt, "Alternatives in digital communications," Proceedings of the IEEE, vol. 61, pp. 703 - 721, Jun. 1973.

[2] A. J. Goldsmith and S. B. Wicker, "Design challenges for energyconstrained ad hoc wireless networks," IEEE Wireless Communications, vol. 9, pp. 8 - 27, Aug. 2002.

[3] R. W. Lucky, J. Salz, and E. J. Weldon, Jr., Principles of Data Communication. New York: McGraw-Hill, 1968.

[4] J. F. Hayes, "Adaptive feedback communications," IEEE Transactions on Communication Technology, vol. 16, pp. 29 - 34, Feb. 1968.

[5] J. K. Cavers, "Variable-rate transmission for Rayleigh fading channels," IEEE Transactions on Communications, vol. 20, pp. 15 - 22, Feb. 1972.

[6] V. O. Hentinen, "Error performance for adaptive transmission on fading channels," IEEE Transactions on Communications, vol. 22, pp. 1331 1337, Sep. 1974.

[7] R. Srinivasan, "Feedback communications over fading channels," IEEE Transactions on Communications, vol. 29, pp. 50 - 57, Jan. 1981.

[8] J. G. Proakis, Digital Communications, 4th ed. New York: McGrawHill, 2001.

[9] A. Chockalingam, P. Dietrich, L. B. Milstein, and R. R. Rao, "Performance of closed-loop power control in DS-CDMA cellular systems," IEEE Transactions on Vehicular Technology, vol. 47, pp. 774789, Aug. 1998.

[10] D. M. Ionescu and A. Boariu, "Predictive closed-loop power control for frequency-division duplex wireless systems," IEEE Communications Letters, vol. 5, pp. 248-250, Jun. 2001.
[11] Wei Xiang and S. S. Pietrobon, "On the capacity and normalization of ISI channels," IEEE Transactions on Information Theory, vol. 49, pp. 2263 - 2268, Sep. 2003.

[12] A. J. Goldsmith and S.-G. Chua, "Variable-rate variable-power MQAM for fading channels," IEEE Transactions on Communications, vol. 45, pp. 1218-1230, Oct. 1997.

[13] I. S. Gradshteyn and I. M. Ryzhik, Table of Integrals, Series, and Products, 5th ed. San Diego, CA: Academic Press, 1994.

[14] I. Saarinen, A. Mämmelä, P. Järvensivu, and K. Ruotsalainen, "Power control in feedback communications over a fading channel," IEEE Transactions on Vehicular Technology, vol. 50, pp. 1231 - 1239, Sep. 2001.

[15] I. Saarinen and A. Mämmelä, "Power control and diversity in feedback communications over a fading channel," IEEE Transactions on Communications, vol. 52, pp. 1857-1861, Nov. 2004. 